\title{
Editorial: Towards Improving the Science of Hormones and Cancer
}

\author{
Carol A. Lange ${ }^{1}$
}

Published online: 24 January 2017

(C) Springer Science+Business Media New York 2017

\section{Dear Readers,}

I am honored to begin anew at Hormones and Cancer as your incoming Editor-in-Chief. As many of you know, I succeeded our founding Editor, Dr. Jonathan J. Li after his passing in 2011. The late Dr. Li was a friend to many in our field who was well known for his warmth and great enthusiasm for science and in particular, as an ally for emerging scientists in training. It was Dr. Li's vision (with the support of the Endocrine Society leadership and Springer) to create a journal that could be a stable venue for the presentation of innovative basic, translational, and clinical cancer research focused on the influence of hormones on cancers of all kinds. By inclusion of a broad range of cancer-focused studies, he sought to bring together scientists across multiple disciplines and working on diverse cancers, but with focus on hormone action. Following my first term (2011-2014) as Editor, Dr. Nancy Weigel (Baylor College of Medicine) served as Editor-in-Chief (2014-2016) alongside her Associate Editors Donald McDonnell (Duke University) and Gary Hammer (University of Michigan). Dr. Weigel's team continued to build and strengthen Hormones and Cancer by featuring authors' work (images of cancer research) on our cover, further focusing our scope and expanding our reach. They contributed to our stability and reputation as a reliable publishing venue for novel studies on hormones and cancer, and I am grateful to Drs. Weigel and McDonnell for agreeing to remain on the editorial board. With the recent departure of the Endocrine Society as our publishing partner, Hormones and Cancer will

Carol A. Lange

lange047@umn.edu

1 Department of Medicine (Division of Hematology, Oncology, and Transplantation) University of Minnesota Masonic Center, Minneapolis, MN, USA

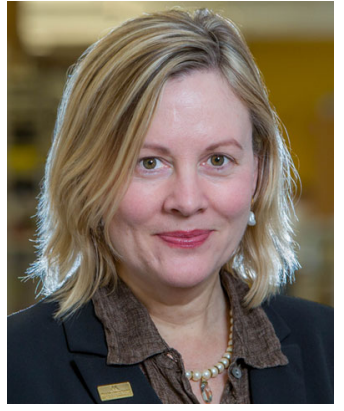

Carol A. Lange, PhD

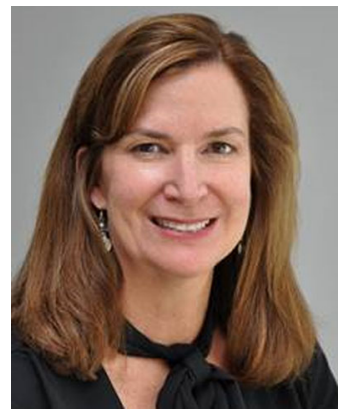

Jennifer K. Richer, PhD

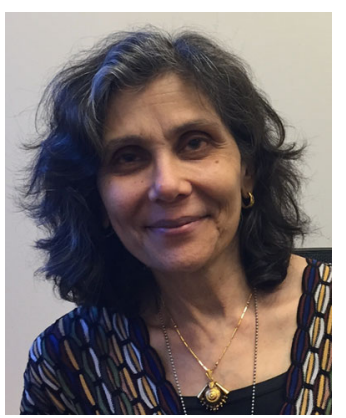

Seema A. Khan, MD, SM 
continue to publish under Springer Nature. Although Hormones and Cancer is still a relatively young journal, we are growing and our 2015 impact factor of 3.167 is predicted to rise.

I am pleased to be joined by two new Associate Editors for Hormones and Cancer, Dr. Jennifer K. Richer, PhD (University of Colorado) and Dr. Seema A. Khan, MD, SM (Feinberg School of Medicine, Northwestern University).

Dr. Richer has over 24 years of experience in the cell and molecular biology of nuclear hormone receptor actions. The Richer laboratory tackles clinically relevant issues related to hormone-dependent women's cancers from a cell and molecular approach. In order to translate her research, Dr. Richer collaborates with oncologists, pathologists, and surgeons. Her recent research demonstrated that breast tumors resistant to anti-estrogen therapy express high levels of nuclear androgen receptor (AR) and that AR can serve as a therapeutic target in both estrogen receptor positive (ER+) and negative disease. As a result of Dr. Richer's preclinical findings, the new generation enzalutamide is currently in clinical trials for both ER+ breast cancer and AR+ triple negative breast cancer (TNBC). Dr. Richer also studies the role of microRNAs differentially expressed in well-differentiated breast and gynecological cancers versus those that have undergone epithelial to mesenchymal transition. Dr. Richer brings considerable basic and translational strength to the journal.

Dr. Seema Ahsan Khan, MD, SM, is a surgical oncologist and clinician scientist working in epidemiology and the identification of novel molecular biomarkers of breast cancer risk, including steroid hormones in breast tissue and nipple fluid. Dr. Khan has considerable expertise in the conduct of early phase prevention trials with biomarker endpoints, ranging from soy isoflavone supplementation in healthy women who are considered to be at high risk for breast cancer development to antiprogesterone compounds and 4-hydroxytamoxifen in the presurgical window setting. She has pioneered the use of novel transdermal delivery methods for breast cancer chemoprevention agents. Dr. Khan's expertise will facilitate seamless review of our preclinical and clinical submissions.

We are proud of our very distinguished Editorial Board, which covers basic science, preclinical, epidemiological, and clinical research with areas of expertise in benign and malignant tumors of hormone secreting endocrine organs as well as the effects of hormones on all types of tumors (see our Aims and Scope). Hormones and Cancer publishes six times per year with an average "time to first decision" of less than 30 days. We are currently soliciting mini-review articles and new research papers in the areas of cancer causation, metabolism, and hormone dependence; disease progression, prevention, treatment, and resistance; translational studies of novel therapies or therapeutic agents; hormone receptors and their co-regulatory molecules; hormonally influenced signaling pathways; nuclear and steroid hormone receptors, adrenal, pituitary, and thyroid hormones as well as vitamin D.

As Hormones and Cancer changes leadership and bids farewell to our Endocrine Society partners, my team and I are motivated to continue in the spirit as advocates for our field. Notably, the recent bipartisan passing of the $\$ 6.3$ billion medical funding legislation known as the $21 \mathrm{st}$ Century Cures Act will include a major boost to cancer research funding through mandatory funding of the recently named Beau Biden Cancer Moonshot. However, as we enter 2017 , the scientific policy and commitment of the new administration to science and medical research is not clear. Now more than ever, we need to be strong advocates for cancer research, as it is clear that fundamental discoveries have translated into high impact new therapies for patients with hormone-dependent cancers. Discoveries of cyclins/cyclin-dependent kinases, signal transduction through PI3K, and steroid hormone synthesis via lyases and aromatases are examples of successful clinical strategies that were first identified in the laboratory. The importance of funding basic science discovery (including emerging technologies) is the cornerstone that ensures our progress towards transformative therapies and reduction of cancer impact. We need to advocate at all levels as citizens (i.e., in speaking to the lay public), as educators, and as mentors of the next generation of scientists and doctors and as active and engaged scientists and physicians who are driven to unravel the complexities of hormone action and hormone-influenced pathways that contribute to health and disease, including cancer and conditions that impact cancer risk. This includes local, national, and international service as leaders in our field. Thus, when called to service as expert scientists (i.e., as mentors, teachers, speakers, authors, reviewers, volunteers, etc.), I urge you to accept. Early in my career following the award of my first R01 (some 20 years ago), I was invited to serve on the Biochemical Endocrinology (BCE) NIH study section. I accepted the challenge and found the panel to be filled with famous names in our field. Although the distinguished membership was initially intimidating, serving alongside these role models inspired me to carefully and responsibly study each grant application and submit comprehensive and objective reviews that were constructive with the goal of improving 
the science. Similarly, as an Editor of scientific content, the goal of the service of publication of innovative research findings is ultimately to improve the science.

Today's funding climate is more competitive than ever. The bar for both authors and reviewers is high. Now more than ever, the most talented scientists are urgently needed to serve not only as peer reviewers but in all types of leadership roles. Active and consistent participation as a field will ensure that quality science, science education, and the development of innovative models, approaches, and technologies remain valued priorities not only of the field of cancer research but of our society as a whole. Today, I am still humbled by the immense talent in our field. With your help, I predict a smooth transition for Hormones and Cancer and continued strong momentum. Please join me in rising to the challenges ahead.

As always, your submissions are welcome in the basic, translational, and clinical sciences of all aspects of hormones and cancer. "Wishing you and yours (as in your families friends and loved ones) and your health and happiness in the New Year.

Regards,

Carol A. Lange, $\mathrm{PhD}$

Editor-in-Chief

Hormones and Cancer 